\title{
¿Perturbation Solution for Four-Stream Infrared Radiative Transfer
}

\author{
JIANGNAN LI \\ Canadian Centre for Climate Modelling and Analysis, Environment and Climate Change Canada, \\ Victoria, British Columbia, Canada \\ HOWARD W. BARKER \\ Environment and Climate Change Canada, Downsview, Ontario, Canada
}

(Manuscript received 4 July 2019, in final form 10 February 2020)

\begin{abstract}
A four-stream solution of the longwave radiative transfer is proposed. It is based on the exact perturbation method utilizing the absorption approximation equation as the zero-order solution. Scattering is handled by the first-order perturbation equation. The two- and four-stream approximations are compared both offline and using data from CALIPSO's dual-wavelength lidar.
\end{abstract}

\section{Introduction}

Scattering of longwave (LW) radiation within Earth's atmosphere is very weak relative to that of solar radiation. As such, climate models have treated, and continue to treat, LW radiative transfer with the absorption approximation (AA), which neglects scattering by cloud and aerosol particles. Observations and theoretical calculations show that when LW scattering is ignored, outgoing LW radiation (OLR) is often overestimated by a few watts per square meter (Fu et al. 1997; $\mathrm{Li}$ and $\mathrm{Fu}$ 2000; Li 2002; Costa and Shine 2006; Joseph and Min 2003; Zhang et al. 2016; Tang et al. 2018). The exact four-stream LW radiative transfer solution was found by Zhang et al. (2016) based on Chandrasekhar's invariance principle. This method is general with no limitation for the strength of scattering. However, the scattering effect in the atmosphere is very weak, as the observations show that the scattering effect only causes a few watts per square meter in ORL compared to the calculations by neglecting scattering (Joseph and Min 2003). Therefore, in climate modeling, the approximation method for weak scattering can be applied. Li (2000) proposed a perturbation method to handle scattering of LW radiation by

๑ Denotes content that is immediately available upon publication as open access.

Corresponding author: Jiangnan Li, jiangnan.li@canada.ca cloud particles, though this method does not exactly follow formal perturbation method in which the zero-order equation should be the AA with scattering added by the first-order perturbation equation. This was done, however, by Li (2002), but only for the two-stream approximation. In this study, the perturbation method for LW scattering is extended to the $2 n$-stream approximation.

It is found that the proposed perturbation method can always produce similar accuracy compared to the exact solution, but the CPU time to execute in the LW radiative transfer solver portion of the code is about two orders faster than the exact solution. This is because the scattering is only calculated the cloud existing layers.

The second section lays down the theoretical basis of the methodology. This is followed by sections devoted to presentation of some results and conclusions.

\section{Theory}

The plane-parallel radiative transfer equation for azimuthally averaged diffuse LW radiance $I(\tau, \mu)$ is

$$
\begin{aligned}
\mu \frac{d I(\tau, \mu)}{d \tau}= & I(\tau, \mu)-\frac{\tilde{\omega}}{2} \int_{-1}^{1} I\left(\tau, \mu^{\prime}\right) P\left(\mu, \mu^{\prime}\right) d \mu^{\prime} \\
& -(1-\tilde{\omega}) B[T(\tau)],
\end{aligned}
$$

where $\mu=\cos \theta, \theta$ is the zenith angle, $\tau$ is the optical depth, $\tilde{\omega}$ is the single-scattering albedo, $B[T(\tau)]$ is the Planck function at temperature $T$, and $P\left(\mu, \mu^{\prime}\right)$ is the azimuthal-independent scattering phase function, which 
describes the probability of radiation incident from direction $\mu$ being scattered into direction $\mu^{\prime}$.

To begin, let $P\left(\mu, \mu^{\prime}\right)$ be represented by the $\delta-M$ approximation (Wiscombe 1977) as

$$
\begin{aligned}
P\left(\mu, \mu^{\prime}\right)= & 2 f \delta\left(\mu-\mu^{\prime}\right)+(1-f)\left[1+\omega_{1} P_{1}(\mu) P_{1}\left(\mu^{\prime}\right)\right. \\
& \left.+\omega_{2} P_{2}(\mu) P_{2}\left(\mu^{\prime}\right)+\cdots\right],
\end{aligned}
$$

where $f$ is fractional scattering into the forward peak, $\omega_{i}$ is the $i$ th moment of $P\left(\mu, \mu^{\prime}\right)$, and $P_{i}(\mu)$ is the $i$ th-order Legendre polynomial. The asymmetry factor is defined as $g=3 \omega_{1}$. For weak scattering and strong absorption, as is usual for LW radiation, scattering can be simplified by setting $f=1$. Thus, scattering is assumed to be entirely into the forward scattering peak (scattering angle is $0^{\circ}$ ). Thus, (1) becomes

$$
\mu \frac{d I(\tau, \mu)}{d \tau}=(1-\tilde{\omega}) I(\tau, \mu)-(1-\tilde{\omega}) B[T(\tau)],
$$

which is the AA, and used currently by almost all climate models, for example, in the RRTM LW radiative solver (Mlawer et al. 1997). To add the scattering effect but retain the simplicity of AA as the primary solution, (1) is rewritten as

$$
\begin{aligned}
\mu \frac{d I(\tau, \mu)}{d \tau}= & \varepsilon\{I(\tau, \mu)-B[T(\tau)]\} \\
& +\tilde{\omega}\left[I(\tau, \mu)-\frac{1}{2} \int_{-1}^{1} I\left(\tau, \mu^{\prime}\right) P\left(\mu, \mu^{\prime}\right) d \mu^{\prime}\right],
\end{aligned}
$$

where $\varepsilon=1-\tilde{\omega}$. Since $\tilde{\omega}<1$, one can use $I(\tau, \mu)=$ $I^{(0)}(\tau, \mu)+\tilde{\omega} I^{(1)}(\tau, \mu)+\cdots$, which when substituted into (4), and retaining just the leading two terms, yields

$$
\begin{gathered}
\mu \frac{d I^{(0)}(\tau, \mu)}{d \tau}=\varepsilon\left\{I^{(0)}(\tau, \mu)-B[T(\tau)]\right\} \\
\mu \frac{d I^{(1)}(\tau, \mu)}{d \tau}=\varepsilon I^{(1)}(\tau, \mu)+I^{(0)}(\tau, \mu) \\
-\frac{1}{2} \int_{-1}^{1} I^{(0)}\left(\tau, \mu^{\prime}\right) P\left(\mu, \mu^{\prime}\right) d \mu^{\prime} .
\end{gathered}
$$

The zero-order solution of (5) is again the AA, with LW scattering addressed in the solution of first-order perturbation of (6).
For nonisothermal layers, it can be assumed that $B$ $[T(\tau)]$ has an exponential dependence on $\tau$ (Fu et al. 1997; Li and Fu 2000). Letting level 1 be the top of the atmosphere (TOA) and level $N$ be the surface, the thermal source term for layer $k$, between levels $k$ and $k+1$, can be written as

$$
B[T(\tau)] \approx B_{k} e^{\beta_{k} \tau / \tau_{k}},
$$

where $\beta_{k}=\ln \left(B_{k+1} / B_{k} s\right)$ with $B_{k}$ being the Planck function evaluated using temperature at level $k$.

Upward and downward radiances at levels $k$ and $k+1$ for the AA are solutions of (5),

$$
I_{k}^{(0)}\left(\mu_{i}\right)=I_{k+1}^{(0)}\left(\mu_{i}\right) e^{-\kappa_{k} / \mu_{i}}+\eta_{k}\left(-\mu_{i}\right)\left(B_{k}-B_{k+1} e^{-\kappa_{k} / \mu_{i}}\right),
$$

$I_{k+1}^{(0)}\left(-\mu_{i}\right)=I_{k}^{(0)}\left(-\mu_{i}\right) e^{-\kappa_{k} / \mu_{i}}+\eta_{k}\left(\mu_{i}\right)\left(B_{k+1}-B_{k} e^{-\kappa_{k} / \mu_{i}}\right)$,

where $\eta_{k}(\mu)=1 /\left(1+\beta_{k} \mu / \varepsilon_{k}\right)$. Since fluxes are defined as integrals of radiances over the up- and down-facing hemispheres, Gaussian quadrature can be used to approximate them. Hence, upwelling flux at level $k$ is

$$
F_{k}^{\uparrow}=2 \pi \int_{0}^{1} I_{k}^{(0)}(\mu) \mu d \mu=2 \pi \sum_{i=1}^{n} I_{k}^{(0)}\left(\mu_{i}\right) \mu_{i} w_{i},
$$

where $w_{i}$ and $\mu_{i}$ are weights and angles, respectively, as determined by the $n$th order of quadrature. The four-stream approximation corresponds to $n=2$ with weights $w_{1}=$ $w_{2}=0.5$ and abscissa of $\mu_{1}=0.21132$ and $\mu_{2}=0.78867$. It is straightforward to extend this to $2 n$-stream approximation using weights and abscissa as listed in $\mathrm{Li}$ (2000).

Correspondingly, upwelling and downwelling radiances at levels $k$ and $k+1$ for the scattering perturbation components are solutions of (6),

$$
\begin{gathered}
I_{k}^{(1)}\left(\mu_{i}\right)=I_{k+1}^{(1)}\left(\mu_{i}\right) e^{-\kappa_{k} / \mu_{i}}+S_{k}^{+}\left(\mu_{i}\right), \\
I_{k+1}^{(1)}\left(-\mu_{i}\right)=I_{k}^{(1)}\left(-\mu_{i}\right) e^{-\kappa_{k} / \mu_{i}}+S_{k}^{-}\left(-\mu_{i}\right),
\end{gathered}
$$

where

$$
\begin{aligned}
S_{k}^{+}\left(\mu_{i}\right)= & -\frac{1}{\varepsilon_{k}} \sum_{j=1}^{n}\left(\psi _ { k } ( \mu _ { i } , \mu _ { j } ) \left\{\mathscr{C}_{k, i, j}\left[I_{k+1}^{(0)}\left(\mu_{j}\right)-\eta\left(-\mu_{j}\right) B_{k+1}\right]\right.\right. \\
& \left.+\eta_{k}\left(-\mu_{j}\right) \eta_{k}\left(-\mu_{i}\right)\left(B_{k}-B_{k+1} e^{-\kappa_{k} / \mu_{i}}\right)\right\} \\
& +\psi_{k}\left(\mu_{i},-\mu_{j}\right)\left\{\frac{1}{\mu_{i} / \mu_{j}+1}\left(1-e^{-\kappa_{k} / \mu_{i}} e^{-\kappa_{k} / \mu_{j}}\right)\left[I_{k}^{(0)}\left(-\mu_{j}\right)-\eta_{k}\left(\mu_{j}\right) B_{k}\right]\right. \\
& \left.\left.+\eta_{k}\left(\mu_{j}\right) \eta_{k}\left(-\mu_{i}\right)\left(B_{k}-B_{k+1} e^{-\kappa_{k} / \mu_{i}}\right)\right\}\right),
\end{aligned}
$$


where $I_{k+1}^{(0)}\left(\mu_{j}\right)$ and $I_{k}^{(0)}\left(-\mu_{j}\right)$ are from (8),

$$
\mathscr{C}_{k, i, j}= \begin{cases}\frac{\kappa_{k}}{\mu_{i}} & i=j, \\ \frac{1}{\mu_{i} / \mu_{j}-1}\left(e^{-\kappa_{k} / \mu_{i}}-e^{-\kappa_{k} / \mu_{j}}\right) & i \neq j,\end{cases}
$$

and

$$
\psi_{k}\left(\mu_{i}, \mu_{j}\right)=\delta_{i, j}-0.5 \sum_{l=0}^{n} \omega_{k, l} P_{l}\left(\mu_{i}\right) P_{l}\left(\mu_{j}\right),
$$

where $\omega_{k, l}$ is the $l$ th moment of $P\left(\mu, \mu^{\prime}\right)$ at layer $k$, and $\delta_{i, j}$ is the Kronecker $\delta$.

It is unnecessary to present $S_{k}^{-}\left(-\mu_{i}\right)$ for downwelling radiance since the physical process is the same as for upwelling. Expressions are the same except that the physical quantities related to "up" and "down" should be exchanged. The general transformations between "up" and "down" are

$$
\begin{aligned}
& I_{k}^{0}(\mu) \leftrightarrow I_{k+1}^{0}(-\mu), F_{k}^{+} \leftrightarrow F_{k+1}^{-}, \\
& B_{k} \leftrightarrow B_{k+1}, \quad \tau_{k} \leftrightarrow-\tau_{k}, \quad \mu_{k} \leftrightarrow-\mu_{k} .
\end{aligned}
$$

Physical quantities that are directionally independent, such as $\omega_{i}$ and $g_{i}$ remain unchanged. All equations regarding "up" and "down" satisfy the relationship of (12). This can be verified in (8), (10), and (13).

From (8) and (10), we have

$$
\begin{aligned}
I_{k}\left(\mu_{i}\right)= & I_{k+1}\left(\mu_{i}\right) e^{-\kappa_{k} / \mu_{i}}+\eta_{k}\left(-\mu_{i}\right)\left(B_{k}-B_{k+1} e^{-\kappa_{k} / \mu_{i}}\right) \\
& +\tilde{\omega}_{k} S_{k}\left(\mu_{i}\right), \\
I_{k+1}\left(-\mu_{i}\right)= & I_{k}\left(-\mu_{i}\right) e^{-\kappa_{k} / \mu_{i}}+\eta_{k}\left(\mu_{i}\right)\left(B_{k+1}-B_{k} e^{-\kappa_{k} / \mu_{i}}\right) \\
& +\tilde{\omega}_{k} S_{k}^{-}\left(-\mu_{i}\right),
\end{aligned}
$$

Equations (8) and (10) are general forms for the $2 n$ stream approximation. Accordingly, (2) can be extended to $\delta-M$ scaling of optical properties (Wiscombe 1977). Upwelling flux at level $k$ can be obtained the same as (9) Hereinafter, four-stream perturbation solutions are denoted as 4SPT, with corresponding two-stream perturbation solutions denoted as 2SPT (Li 2002).

For AA there are two calculation paths: one each for downwelling and upwelling radiances [see (8)]. For the perturbation method, the number of calculation paths increases to four: two for zero order [using (8)] and two for the scattering effect [using (13)]. The number of calculation paths can, however, be reduced to three as follows. First, use (8b) to obtain zero-order downwelling radiance $I_{k}^{(0)}\left(-\mu_{i}\right)$. Then calculate upwelling radiance $I_{k}\left(\mu_{i}\right)$ based on (13a). For $S_{k}\left(\mu_{i}\right)$ in (13a), $I_{k}^{(0)}\left(-\mu_{i}\right)$ from (8b) is used for downwelling radiance, while for upwelling radiance $I_{k+1}\left(\mu_{i}\right)$ is used instead of $I_{k+1}^{(0)}\left(\mu_{i}\right)$. This is because upwelling radiance is calculated layer by layer, and $I_{k+1}\left(\mu_{i}\right)$ is already known from $I_{k}\left(\mu_{i}\right)$, thus saving one calculation path. Finally, calculate downwelling radiance again based on (13b) to include the scattering effect using $I_{k}\left(-\mu_{i}\right)$ and $I_{k+1}\left(\mu_{i}\right)$ to calculate $S_{k}^{-}\left(-\mu_{i}\right)$. This strategy leads to negligible errors. As such, all subsequent calculations for both 2SPT and 4SPT are based on it.

The advantage of the perturbation method is that $S_{k}\left(\mu_{i}\right)$ and $S_{k}\left(\mu_{i}\right)$ need be calculated for only layers with clouds. This makes the perturbation method very efficient.

\section{Results}

The basic radiative transfer model used here is also used in the Fourth Generation Canadian Atmospheric Global Climate Model (CanAM4) (von Salzen et al. 2013). It is based on the correlated- $k$ distribution (CKD) method $\mathrm{Li}$ and Barker (2005) for estimating gaseous transmittances for $\mathrm{H}_{2} \mathrm{O}, \mathrm{O}_{3}, \mathrm{CH}_{4}, \mathrm{CO}_{2}, \mathrm{~N}_{2} \mathrm{O}, \mathrm{CO}, \mathrm{O}_{2}$, and CFC. Nine LW bands are adopted in the wavenumber ranges 2200-2500, 2200-1900, 1400-1900, 1100-1400, 980-1100, 800-980, $540-800,340-540$, and $0-340 \mathrm{~cm}^{-1}$. Cloud particle optical properties vary slowly with wavenumber; thus, parameterizations of aerosol and cloud optical properties in CanAM4 are appropriately weighted mean values over each wavenumber band. Cloud optical properties are parameterized as functions of particle size and cloud water content (Lindner and Li 2000; Yang et al. 2015).

First, consider standalone application of the models to the midlatitude summer (MLS) profile. Zhang et al. (2016) proposed an exact four-stream solution for LW radiative transfer based on Chandrasekhar's invariance principle. It is denoted here as 4DDA. To highlight the accuracy of 4SPT, results for both 4DDA and 2SPT are included in this subsection. Benchmark results come from the 128-stream DISORT model (Stamnes et al. 1988).

The top two rows of Fig. 1 show flux errors as functions of altitude for clear-sky conditions relative to 128-stream DISORT. The clear-sky case has no scattering; thus, the errors arise only from how the integral over the zenith angle is discretized. The 2SPT produces relatively large errors for downwelling flux, especially in bands 5-8, which cover the atmospheric window region. At the surface, these errors reach $0.5 \mathrm{~W} \mathrm{~m}^{-2}$. The two four-stream schemes show very similar results that are superior to those of the 2SPT for all bands. To some extent, however, positive and negative errors cancel one another. This is evident in the rightmost plots, which 
Error in Downwelling Flux $\left(\mathrm{W} \mathrm{m}^{-\mathrm{z}}\right)$
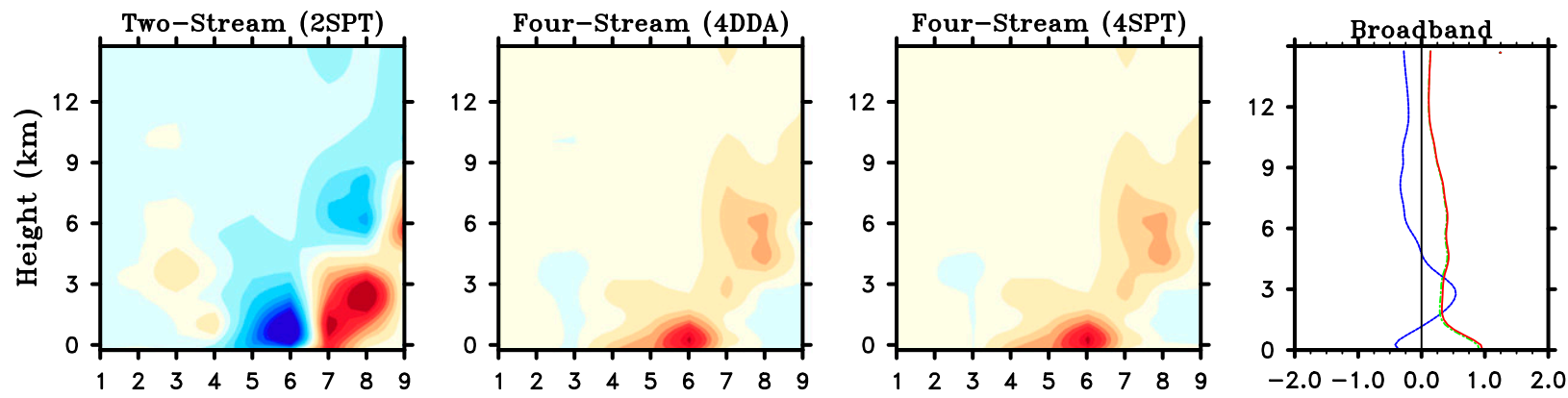

Error in Upwelling Flux $\left(\mathrm{W} \mathrm{m}^{-2}\right)$
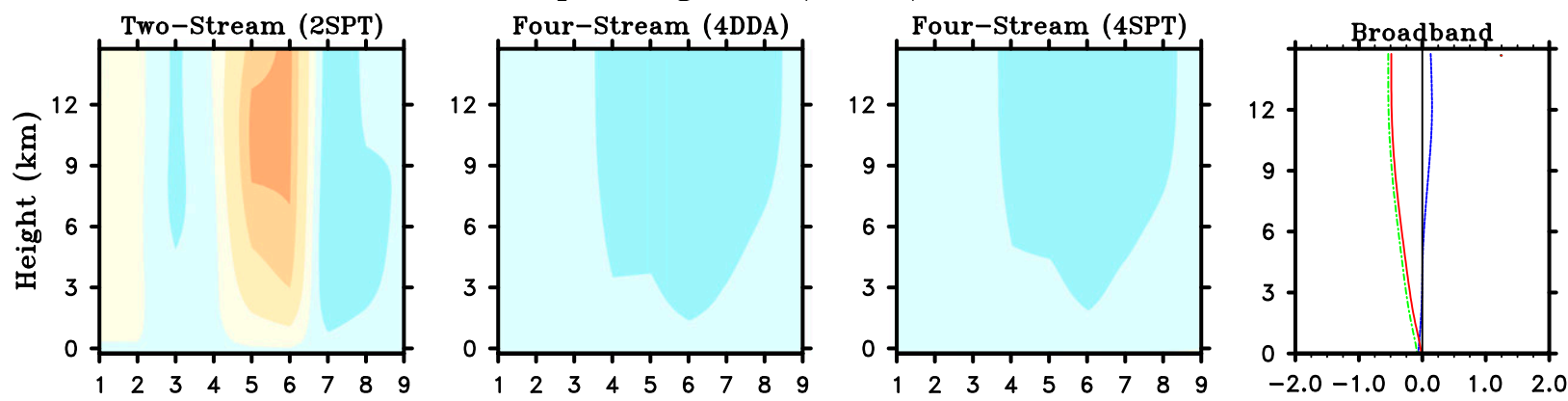

Error in Downwelling Flux $\left(\mathrm{W} \mathrm{m}^{-2}\right)$
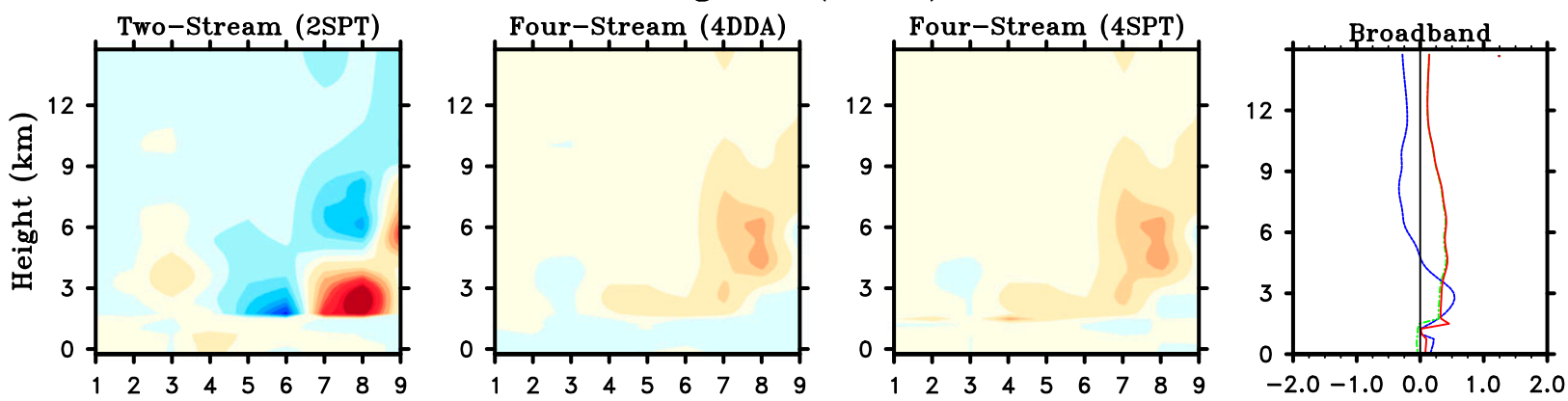

Error in Upwelling Flux $\left(\mathrm{W} \mathrm{m}^{-2}\right)$
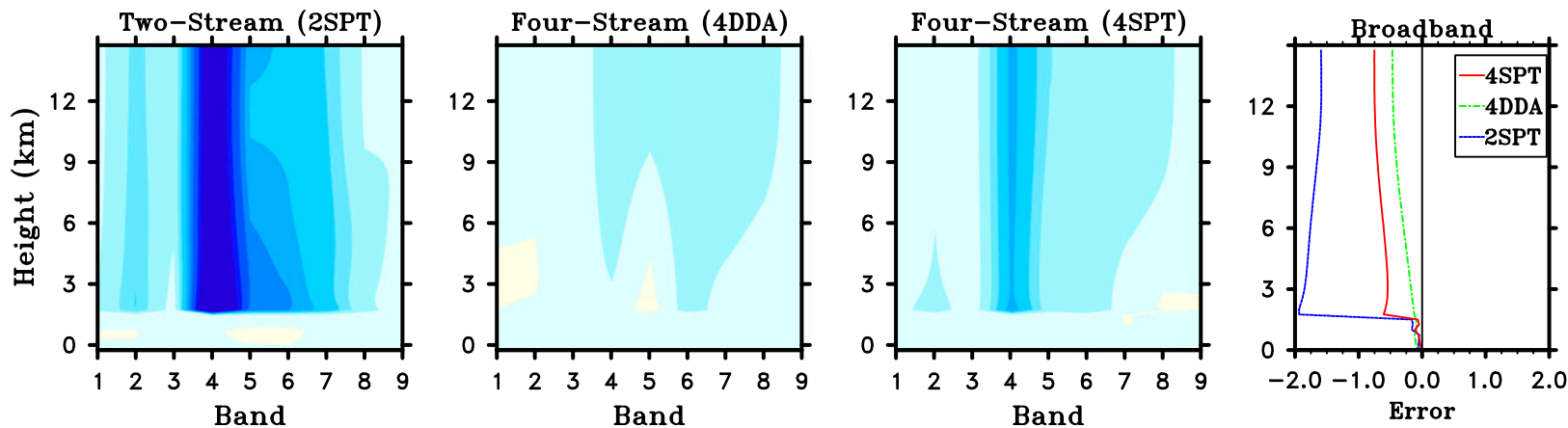

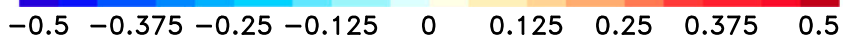

FIG. 1. (top two rows) Errors in LW downwelling and upwelling fluxes for midlatitude summer (MLS) clear-sky conditions for (left to right) 2SPT, 4DDA, 4SPT, and broadband results. (bottom two rows) As in the top two rows, but with a low cloud. Benchmark results were generated by a 128-stream version of DISORT. 
show profiles of spectral-integrated errors. For upward integrated flux, 4SPT is inferior to 2SPT due to cancellation of positive and negative errors for the latter. Nevertheless, clear-sky upwelling flux is over $300 \mathrm{~W} \mathrm{~m}^{-2}$, so errors smaller than $0.5 \mathrm{~W} \mathrm{~m}^{-2}$ will impact climate model simulations minimally.

The bottom two rows of plots in Fig. 1 show flux errors for cloudy conditions with the MLS profile. In this case, the cloud is uniform, overcast, and liquid and located between 1 and $2 \mathrm{~km}$ with liquid water content (LWC) $0.2 \mathrm{~g} \mathrm{~m}^{-3}$ and effective radius $R_{e}$ of $5.89 \mu \mathrm{m}$ (Lindner and $\mathrm{Li}$ 2000). Above the cloud downwelling fluxes are the same as those for the clear sky. Below cloud, downwelling fluxes are much reduced and errors also become smaller compared to the clear-sky case. For upwelling fluxes above the cloud, 2SPT shows a relatively large error in band 4. A similar result is found in 4SPT, though much smaller in magnitude. In this case, 4DDA produces the best results.

Figure 2 shows results for two additional cloudy conditions, again using the MLS profile. The top two rows correspond to a uniform overcast ice cloud located between 9 and $11 \mathrm{~km}$ with an ice water content (IWC) of $0.0048 \mathrm{~g} \mathrm{~m}^{-3}$ and an effective crystal size of $41.5 \mu \mathrm{m}$ (Yang et al. 2015). Note that effective diameter (DG) is double the effective crystal size. Since temperature differences between surfaces and high clouds are usually large, high clouds often have large impacts on LW fluxes. For the 2SPT, large errors occur in bands 5-8 for downwelling fluxes, but with positive and negative errors partly cancelling below $\sim 4 \mathrm{~km}$. Likewise, large errors occur for upwelling flux above cloud base. Both four-stream methods exhibit comparable errors for downwelling and upwelling fluxes, which are much smaller than corresponding errors for the 2SPT. Spectrally integrated errors for 2SPT and 4SPT are comparable.

The bottom two rows in Fig. 2 show results for a more complicated cloudy case: low and middle clouds located between 1-2 and 4-5 km, with LWCs of 0.2 and $0.28 \mathrm{~g} \mathrm{~m}^{-3}$, and $R_{e}$ of 5.89 and $6.2 \mu \mathrm{m}$, respectively. Above them is a high cloud identical to the one used in the previous example. For downwelling flux, large errors for 2SPT that occurred below the solitary high cloud are now attenuated by the middle cloud, but build up downward, only to repeat the pattern once the low cloud is encountered. Again, results for the fourstream schemes are similar and much better than those for the 2SPT. For upwelling flux, the large errors in 2SPT above the middle cloud are suppressed by the high cloud, which leads to upwelling fluxes being much more accurate than for the case of an isolated high cloud. The spectrally integrated upwelling flux errors for 2SPT are smaller than those for 4SPT for due to patterns of positive and negative errors across the spectrum.

Figure 3 shows corresponding heating-rate errors for three cloudy cases presented in Figs. 1 and 2. For low cloud only, 4DDA and 4SPT produce very similar results, both being much more accurate than 2SPT. For high cloud only, 2SPT produces large errors at cloud top for bands 4-9. For the three-cloud case, fairly large errors persist at the top and bottom of each cloud. The four-streams affect dramatic improves for all three cloud scenarios. As with fluxes, differences between the two four-stream models are trivial. In contrast to results for fluxes, 4SPT's heating rate errors are usually significantly smaller than those for 2SPT.

Consider now radiative errors for wider ranges of cloud properties. The top two rows of Fig. 4 show upwelling flux errors at TOA and downwelling flux errors at the surface (SFC) for the low-cloud case presented in Fig. 1. In each panel, $R_{e}$ varies horizontally and liquid water path (LWP; the vertical integral of LWC) varies vertically. Results are shown for 2SPT, 4SPT, and AA. Since only the two stream is used for AA, it is denoted as 2SAA. Results for 2SAA are very similar to those using the four-stream AA as both lack scattering effects ( $\mathrm{Li}$ and $\mathrm{Fu} 2000$ ). Also, since results for 4DDA and 4SPT are very similar, only the 4 SPT results are presented.

For the low cloud, 2SAA overestimates upwelling flux at TOA (also known as outgoing LW radiation or OLR) because no upwelling $\mathrm{LW}$ radiation gets reflected back toward SFC. These errors can exceed $5 \mathrm{~W} \mathrm{~m}^{-2}$. Accuracies are much improved for 2SPT; the maximum error reaches $2 \mathrm{~W} \mathrm{~m}^{-2}$ when $R_{e}$ is small, which is consistent with Fig. 1 for $R_{e}=5.89 \mu \mathrm{m}$. Errors decrease as $R_{e}$ increases since the scattering effect becomes weaker. Accuracy is further improved for 4SPT where absolute errors are limited to $1 \mathrm{~W} \mathrm{~m}^{-2}$. For downwelling flux at SFC, errors are much smaller than those for OLR for all schemes. Conversely, errors become larger when LWP is small as more radiative energy can penetrate through clouds.

The bottom two rows of Fig. 4 show errors for OLR and downwelling flux at SFC for the high-cloud case presented in Fig. 2. Variations of DG and IWP are considered. Again, 2SAA overestimates OLR by over $10 \mathrm{~W} \mathrm{~m}^{-2}$ for small DG, though errors vary dramatically with different DG and IWP. This is consistent with observations, that indicate that scattering effects are very different for different cloud conditions (Joseph and Min 2003). Accuracy is much improved by 2SPT with absolute errors less than $1.5 \mathrm{~W} \mathrm{~m}^{-2}$ for most conditions. Absolute errors for 4SPT are limited to $1.5 \mathrm{~W} \mathrm{~m}^{-2}$ except when DG is small. As shown in Fig. 2, OLR for 
Error in Downwelling Flux $\left(\mathrm{W} \mathrm{m}^{-\mathrm{z}}\right)$
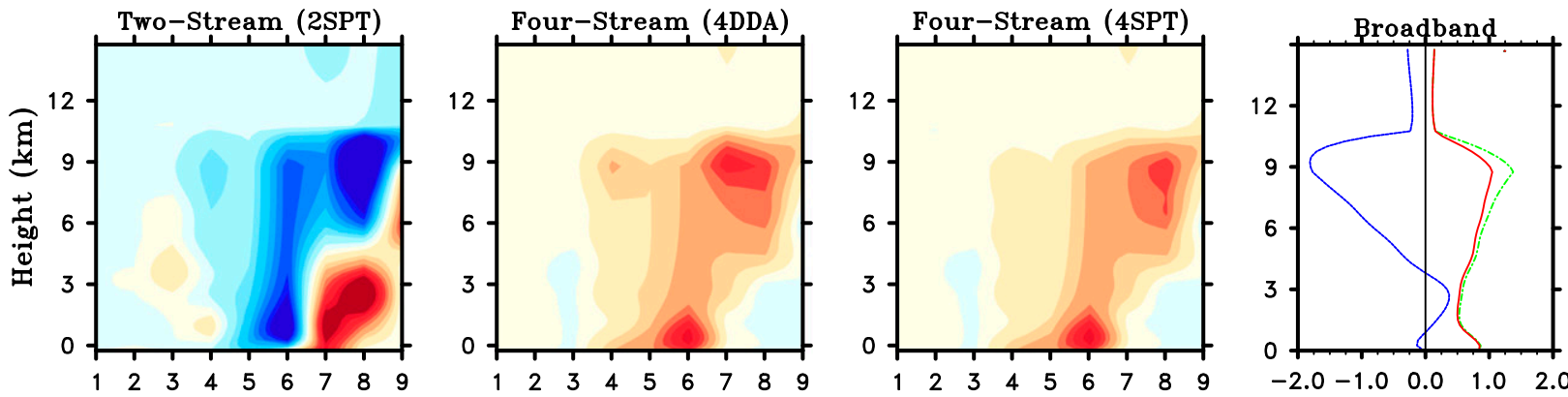

Error in Upwelling Flux $\left(\mathrm{W} \mathrm{m}^{-2}\right)$
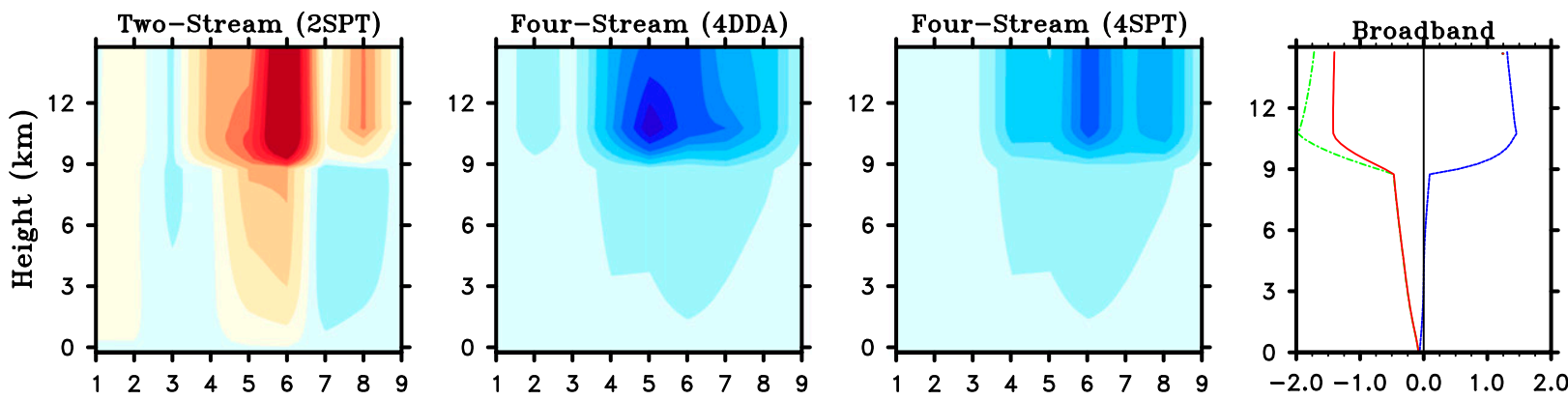

Error in Downwelling Flux $\left(\mathrm{W} \mathrm{m}^{-2}\right)$
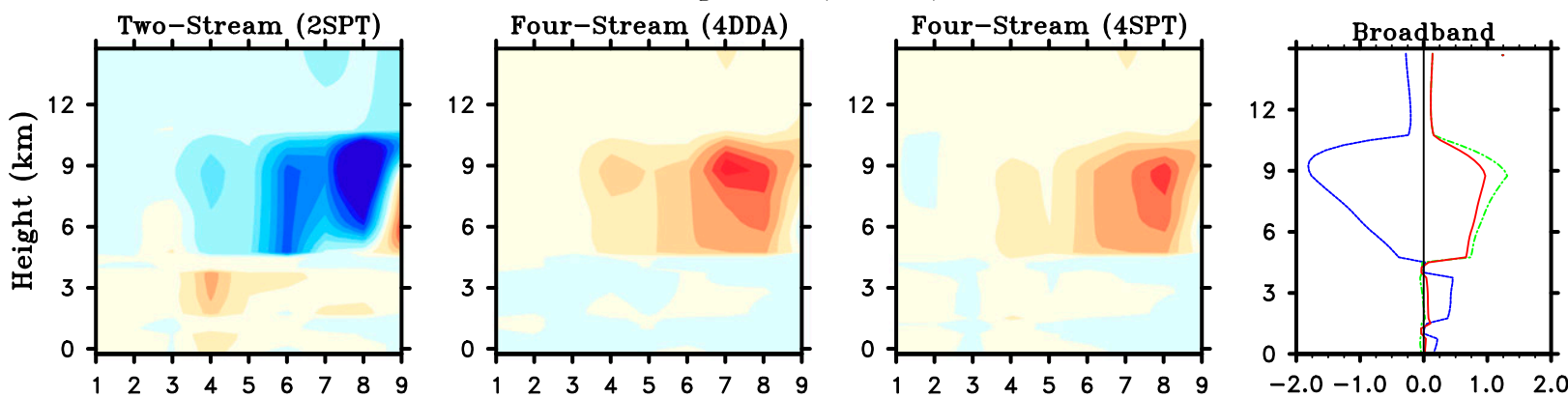

Error in Upwelling Flux $\left(\mathrm{W} \mathrm{m}^{-2}\right)$
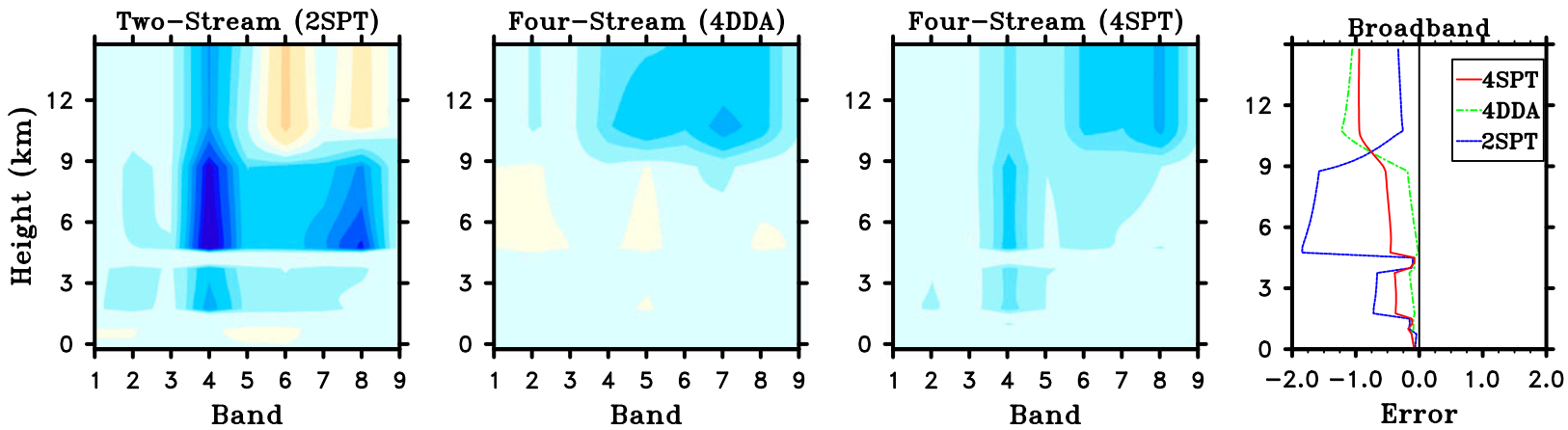

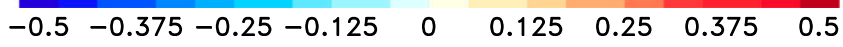

FIG. 2. As in Fig. 1, but for the MLS atmosphere with (top two rows) a high cloud and (bottom two rows) low, middle, and high clouds. 
Error in Heating rate $(\mathrm{K} /$ day)
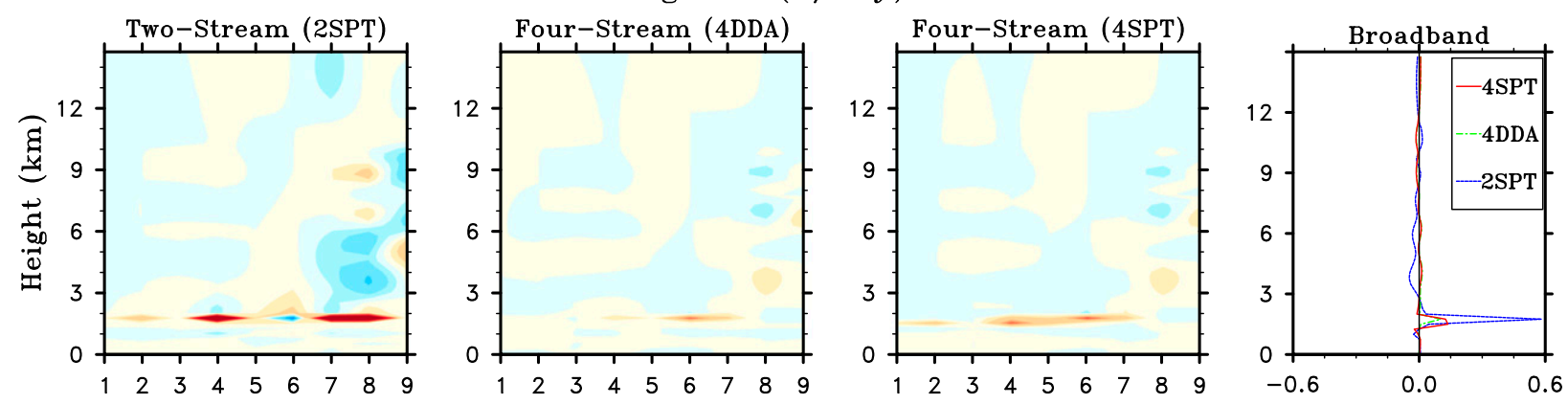

Error in Heating rate (K/day)
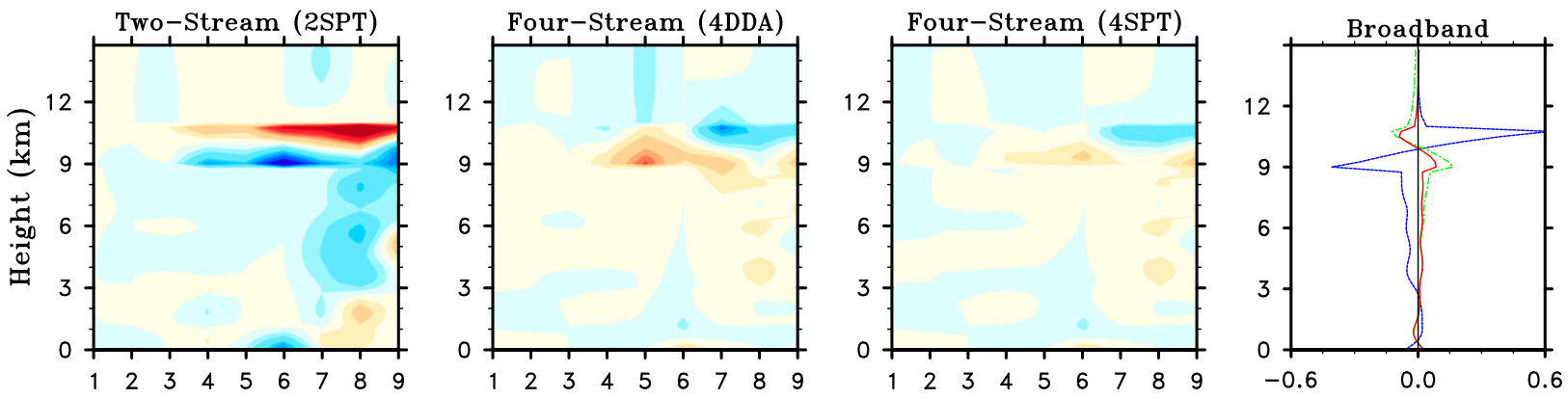

Error in Heating rate (K/day)
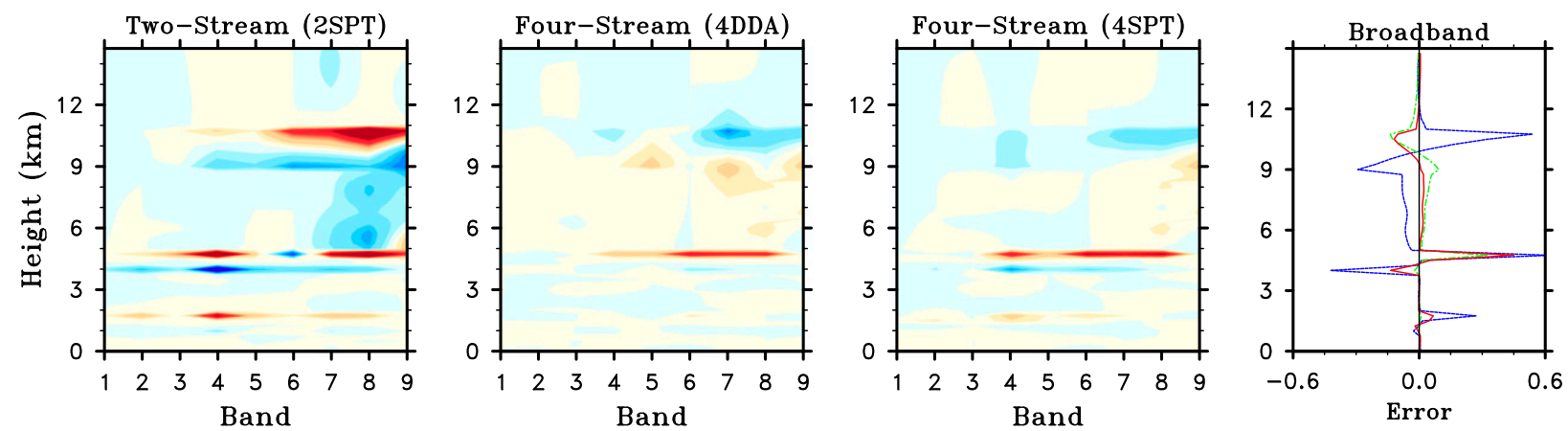

$$
\begin{array}{lllll}
-0.1 & -0.05 & 0 & 0.05 & 0.1
\end{array}
$$

FIG. 3. As in Figs. 1 and 2, but showing LW heating rates for (top) the low-cloud case, (middle) the high-cloud case, and (bottom) the case with three cloud layers.

4SPT is inferior to that of 2SPT. The reason for this is cancellation of spectral positive and negative errors. Errors for downwelling flux at SFC for 2SAA can exceed $5 \mathrm{~W} \mathrm{~m}^{-2}$. These are improved considerably by $2 \mathrm{SPT}$. On the other hand, 4SPT produces absolute errors of 0.75 $1 \mathrm{~W} \mathrm{~m}^{-2}$, and so is inferior to 2SPT. This is consistent with Fig. 2. Downwelling fluxes in high-cloud cases are usually several hundred watts per square meter; the relative error of $4 \mathrm{SPT}$ is always less than $0.5 \%$.

Figure 5 shows the three-cloud case, as used for Fig. 2, but with variations of $R_{e}$ (DG) and LWP (IWP). In the top row, $R_{e}$ and DG are as in Fig. 2, but LWP and IWP vary. For OLR, 2SAA's errors exceed $6 \mathrm{~W} \mathrm{~m}^{-2}$. Both 2SPT and 4SPT limit corresponding errors to less than $1 \mathrm{~W} \mathrm{~m}^{-2}$. Compared to 2SPT, 4SPT produces better results for small LWP and a poorer result for larger LWP. Errors for downwelling flux at SFC are not shown as they are much smaller than those for OLR (cf. Fig. 2). In the bottom row of Fig. 5 LWP and IWP are as in Fig. 2, but $R_{e}$ and DG were allowed to vary. Low and middle clouds had the same $R_{e}$. Again, 2SAA is very poor and produces errors over $10 \mathrm{~W} \mathrm{~m}^{-2}$. For most conditions, 
Error in Upwelling Flux at TOA $\left(\mathrm{Wm}^{-2}\right)$
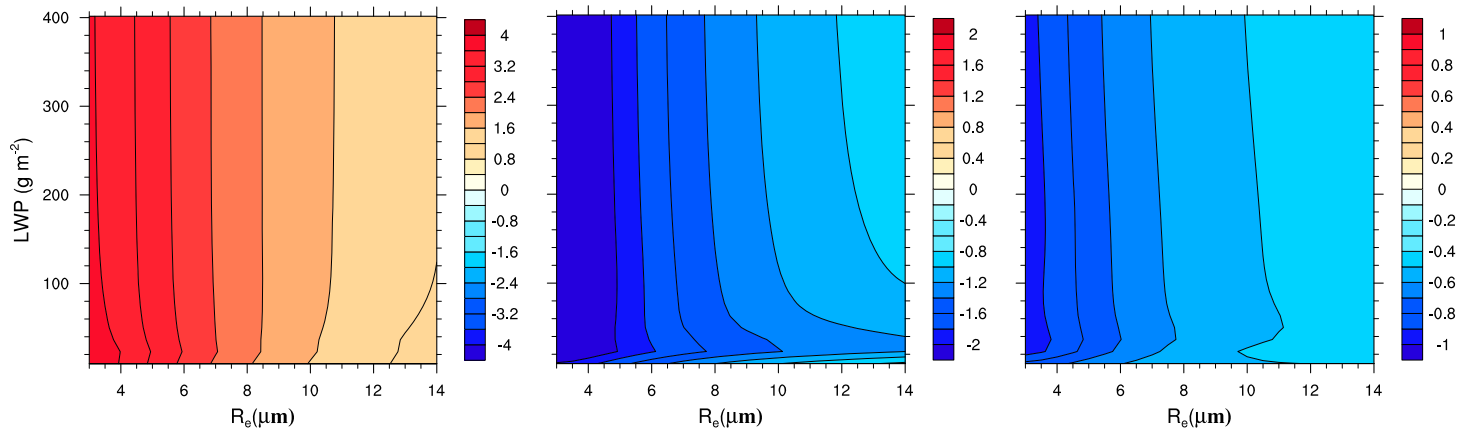

Error in Downwelling Flux at SFC $\left(\mathrm{Wm}^{-2}\right)$
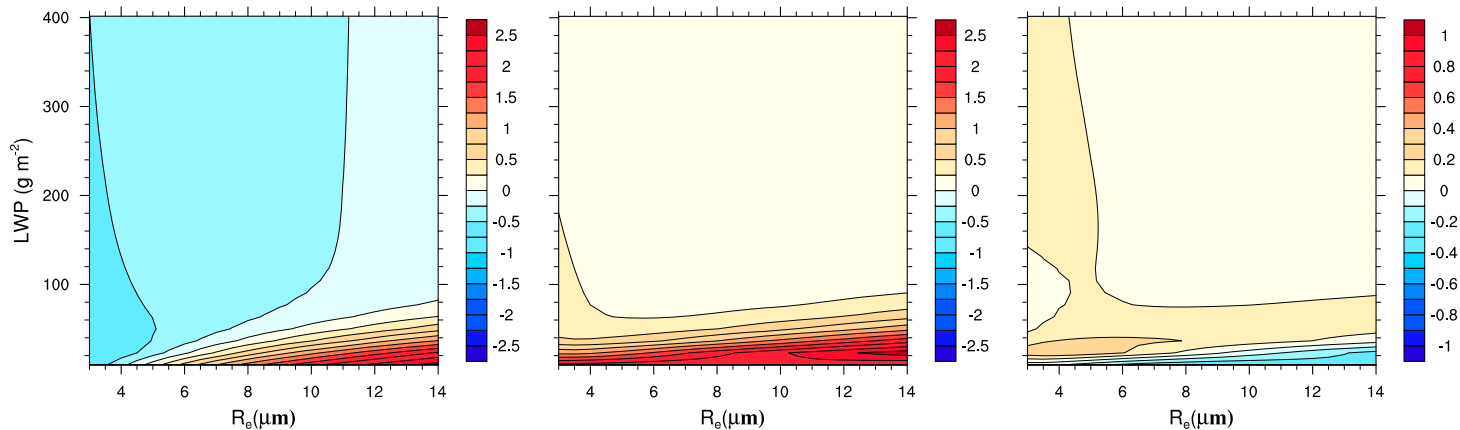

Error in Upwelling Flux at TOA $\left(\mathrm{Wm}^{-2}\right)$
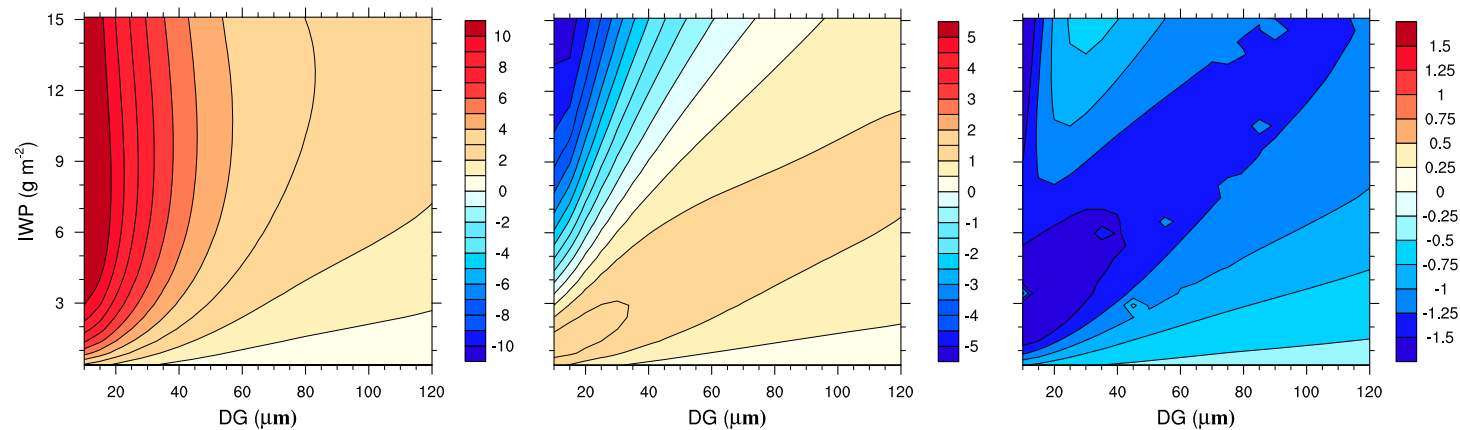

Error in Downwelling Flux at $\mathrm{SFC}\left(\mathrm{Wm}^{-2}\right)$
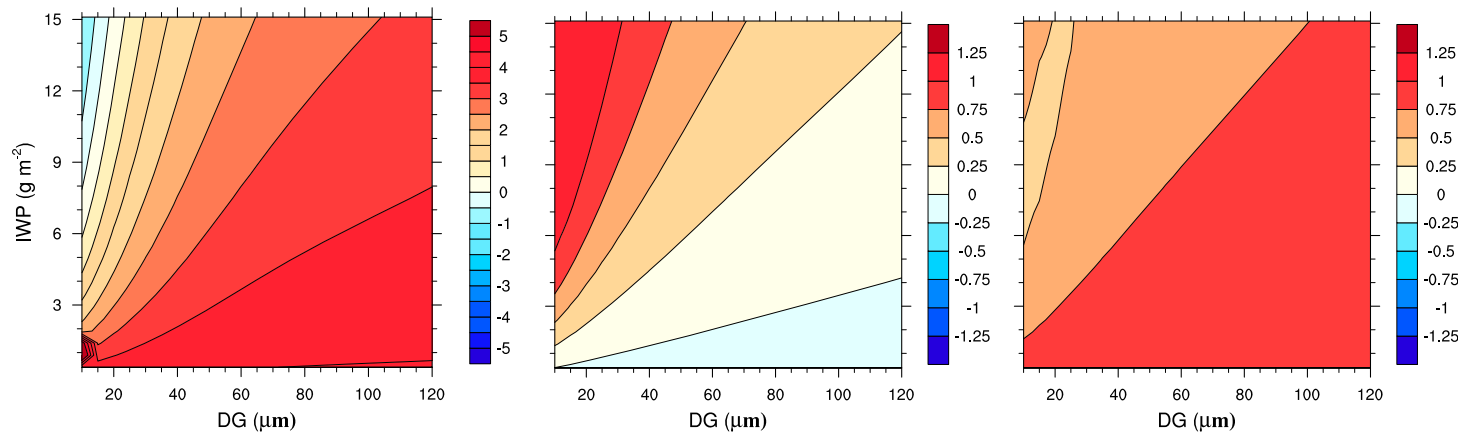

FIG. 4. (top two rows) Errors in LW upwelling flux at TOA and downwelling flux at $\mathrm{SFC}$ for the low-cloud case shown in Fig. 1, with variations of $R_{e}$ and LWP for (left) 2SAA, (center) 2SPT, and (right) 4SPT. (bottom two rows) As in the top two rows, but for the high-cloud case shown in Fig. 2, with variations of DG and IWP. Benchmark results were generated by a 128-stream version of DISORT. 
2SAA

2SPT

$4 \mathrm{SPT}$
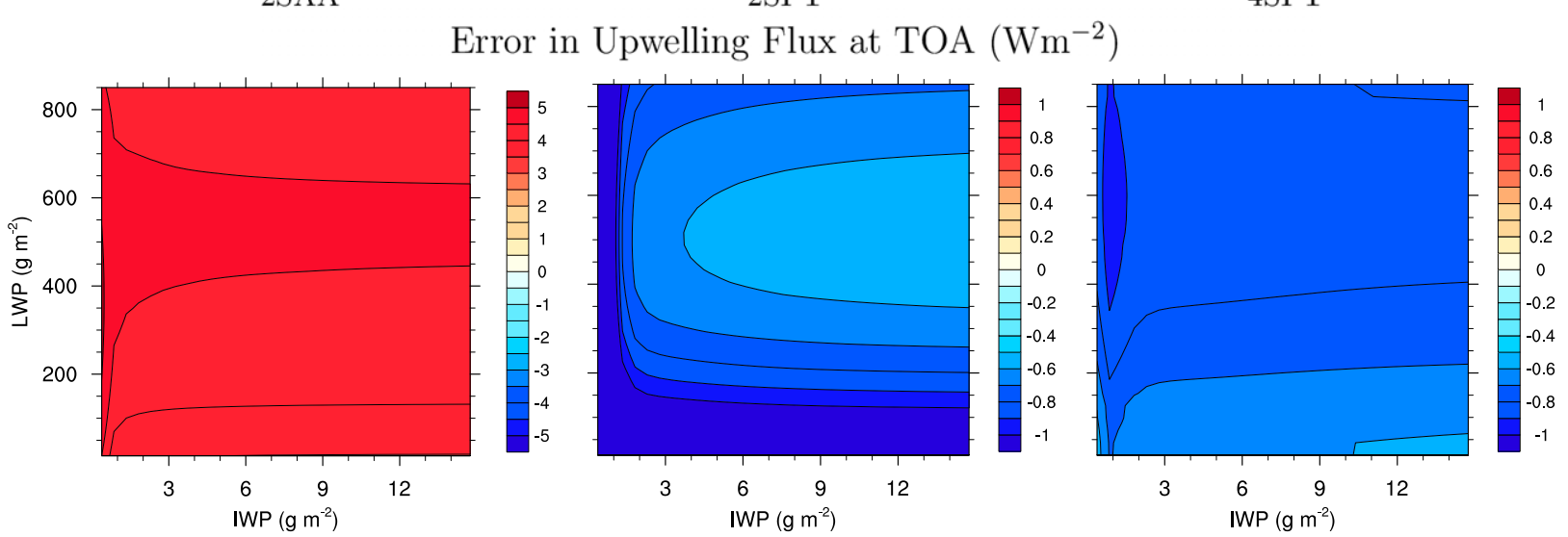

Error in Upwelling Flux at TOA $\left(\mathrm{Wm}^{-2}\right)$
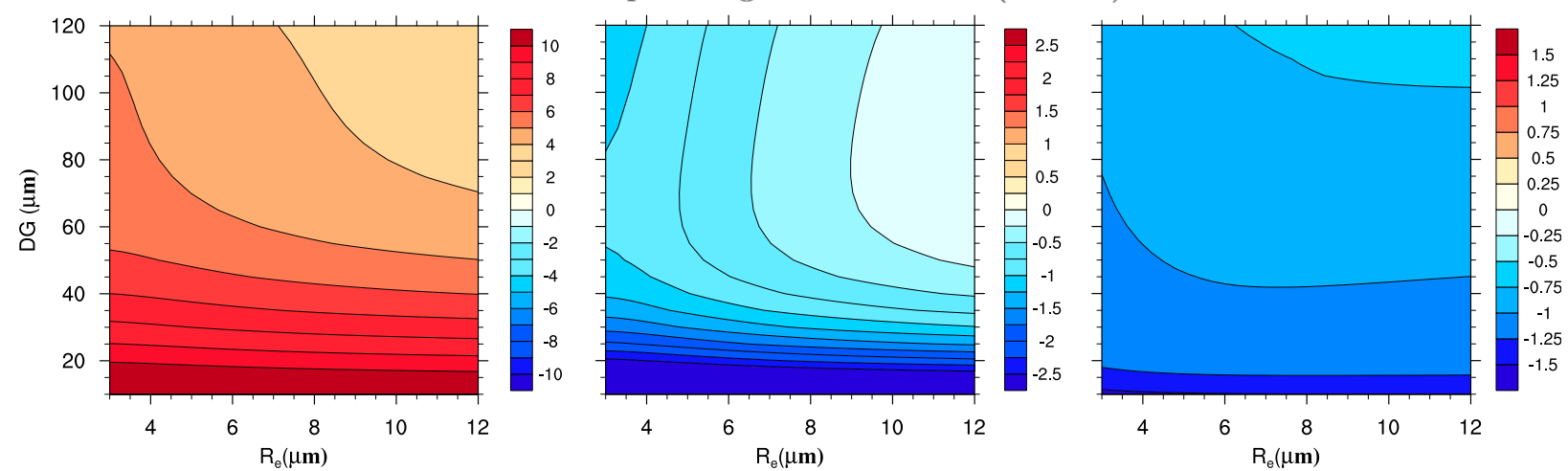

FIG. 5. Errors in upwelling flux at TOA for (left) 2SAA, (center) 2SPT, and (right) 4SPT. (top) Clouds have the same $R_{e}$ and DG as in Fig. 2, but LWP and IWP vary as shown. (bottom) Clouds have the same LWP and IWP as in Fig. 2, but $R_{e}$ and DG vary as shown.

2SPT and 4SPT are comparable. For small DG errors for 2SPT reach almost $3 \mathrm{~W} \mathrm{~m}^{-2}$, but 4 SPT restricts errors to less than $1.75 \mathrm{~W} \mathrm{~m}^{-2}$.

To further verify accuracies of 2SPT and 4SPT, domains with cloud properties were retrieved from measurements made by CALIPSO's dual-wavelength lidar (Winker et al. 2003) and CloudSat's 94-GHz cloudprofiling radar (Stephens et al. 2002). Estimates of cloud liquid and ice water contents can be found in CloudSat's 2B-GEOPROF lidar data (Mace et al. 2007). Each layer is $0.24 \mathrm{~km}$ thick with the lowest two or three layers being unworkable due to ground-clutter contamination. Columns are $1.2 \mathrm{~km}$ wide. CloudSat's ECMWF-AUX files report profiles of pressure, temperature, and mixing ratios of water vapor and ozone for each column. See Barker (2008) for additional details. Assuming that GCM grid columns are nominally $250 \mathrm{~km}$ across, CloudSat-CALIPSO data were partitioned into $256-\mathrm{km}$ segments. Only segments with cloud fraction $>0.05$ were used (Barker et al. 2015). This resulted in 3026 domains distributed across all latitudes for August 2007. For each domain, profiles of cloud fraction, mean and variance of cloud water content were computed for each domain and used by a stochastic cloud generator (Raisanen et al. 2004), which produced 100 subcolumns per domain per radiation calculation.

Figure 6 presents errors for OLR and downwelling flux at SFC for the 3026 domains across all latitudes. Consistent with the previous idealized cases, 2SAA overestimates the OLR, especially in the subtropics where errors often exceed $4 \mathrm{~W} \mathrm{~m}^{-2}$. Corresponding errors for 2 SPT and 4SPT are usually close to -1 and $-0.5 \mathrm{~W} \mathrm{~m}^{-2}$, respectively. For downwelling flux at SFC, errors for 2SAA can close often to zero but can reach $2 \mathrm{~W} \mathrm{~m}^{-2}$. Those for $2 \mathrm{SPT}$ and $4 \mathrm{SPT}$, however, are also mostly near zero but very occasionally exceed $-1 \mathrm{~W} \mathrm{~m}^{-2}$. These are consistent with results shown in Fig. 4.

\section{Conclusions}

In conclusion, a general solution of LW radiative transfer was presented following the standard perturbation method: zero-order solution being the absorption 
Error in Upwelling Flux at TOA $\left(\mathrm{Wm}^{-2}\right)$
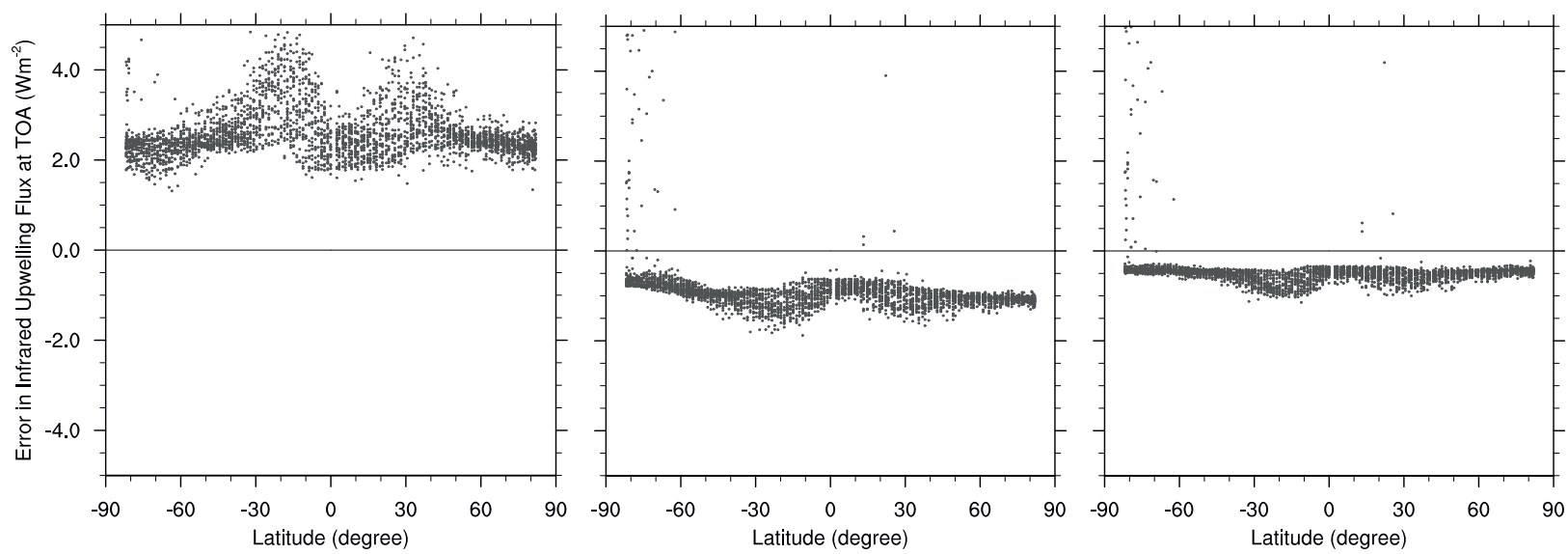

Error in Downwelling Flux at SFC $\left(\mathrm{Wm}^{-2}\right)$
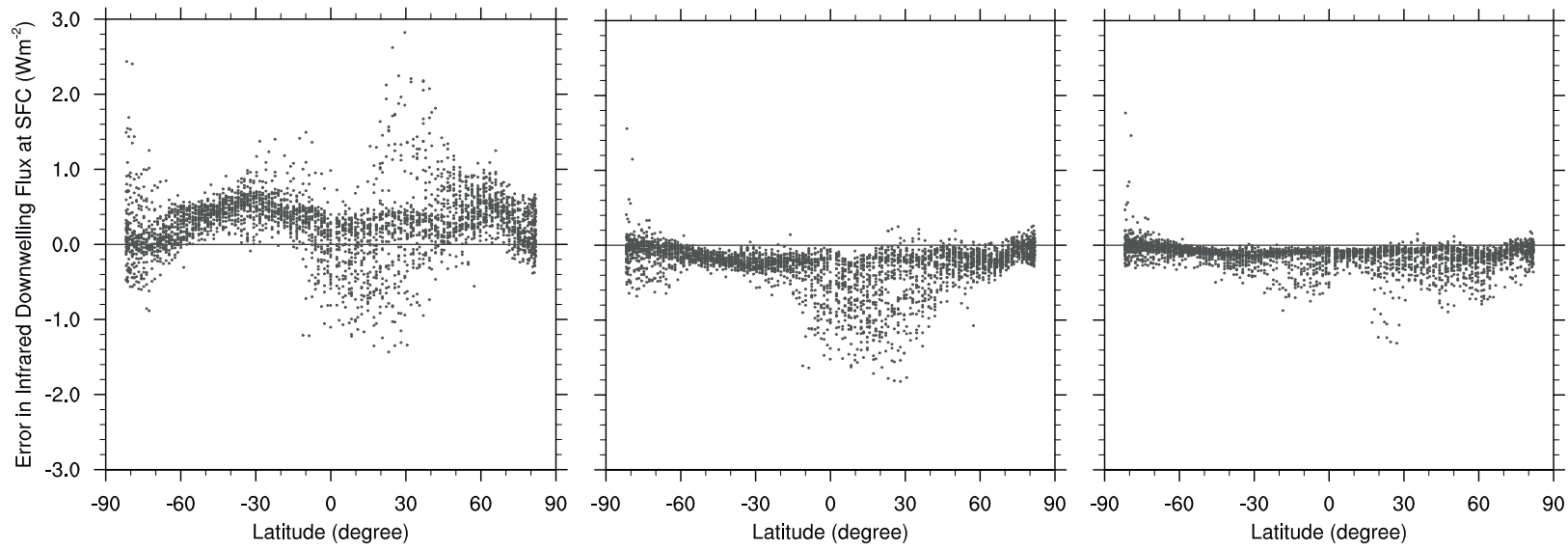

FIG. 6. Errors in (top) upwelling flux at TOA and (bottom) downwelling flux at SFC for (left) 2SAA, (center) 2SPT, and (right) 4SPT. Each point represents mean results for 100 statistically generated subcolumns based on cloud properties derived from data of $C l o u d S a t-$ CALIPSO 256-km domains.

approximation (as used in many dynamical models), with scattering accounted for by a first-order perturbation solution. When the four-stream approximation is used this method has accuracies that are comparable to its corresponding exact solution based on Chandrasekhar's invariance principle; 4DDA. 4SPT is, however, $\sim 100$ times faster than 4DDA. For 4DDA there is no limitation for the strength of scattering, but 4SPT is only applicable to the weak-scattering case. The comparisons show that the weak-scattering condition is satisfied for atmospheric LW radiation. Compared to a 128-stream approximation, 4SPT's errors are almost always smaller than those for its 2-stream counterpart, especially heating-rate errors. Compared to 2SPT, 4SPT requires approximately $50 \%$ more CPU time to execute in the LW radiative transfer solver portion of the code. Since the LW radiative transfer solver uses less than $10 \%$ of the total computing time of the radiation algorithm Li et al. (2005) using 2SPT, the extra CPU time for 4SPT leads to only $5 \%$ total CPU time. Moreover, it is found that flux errors at TOA and SFC do not always correspond to errors within the atmosphere; the latter can be much larger. Also, spectral errors can cancel substantially once broadband integrals are taken, which happens in 2SPT. Last, the formulas presented in this study are general and can be used for $N$-stream approximations.

Given that atmospheric temperatures are usually 200-350 K, most LW energy resides in the atmospheric window range. The fact that single scattering albedo $\tilde{\omega}$ 
for most clouds for wavelengths longer than $8 \mu \mathrm{m}$ is less than 0.7 (Lindner and Li 2000; Yang et al. 2015) explains why the first-order perturbation method works well, as shown in the text. On the other hand, in hightemperature environments, such as combustion systems where temperatures can exceed $1000 \mathrm{~K}$, most radiant energy is near wavelengths of $1 \mu \mathrm{m}$ (Viskanta and Menguc 1987); thus, $\tilde{\omega}$ can be closer to 1 , since $\tilde{\omega}$ increases with decrease of wavelength. In such cases, either higher-order perturbation terms or exact solutions (Zhang et al. 2016) might be required.

Acknowledgments. The authors appreciate two anonymous reviewers for their constructive comments. The codes of 2SPT and 4SPT are available from the authors.

\section{REFERENCES}

Barker, H., 2008: Overlap of fractional cloud for radiation calculations in GCMS: A global analysis using CloudSat and CALIPSO data. J. Geophys. Res., 113, D00A01, https:// doi.org/10.1029/2007JD009677.

_ _ J. Cole, and J. Li, 2015: Application of a Monte Carlo solar radiative transfer model in the McICA framework. Quart. J. Roy. Meteor. Soc., 141, 3130-3139, https://doi.org/10.1002/ qj.2597.

Costa, S. M. S., and K. P. Shine, 2006: An estimate of the global impact of multiple scattering by clouds on outgoing longwave radiation. Quart. J. Roy. Meteor. Soc., 132, 885-895, https:// doi.org/10.1256/qj.05.169.

Fu, Q., K. N. Liou, M. C. Cribb, T. P. Charlock, and A. Grossman, 1997: Multiple scattering parameterization in thermal infrared radiative transfer. J. Atmos. Sci., 54, 2799-2812, https://doi.org/ 10.1175/1520-0469(1997)054<2799:MSPITI>2.0.CO;2.

Joseph, E., and Q. Min, 2003: Assessment of multiple scattering and horizontal inhomogeneity in IR radiative transfer calculations of observed thin cirrus clouds. J. Geophys. Res., $\mathbf{1 0 8}$, 4380, https://doi.org/10.1029/2002JD002831.

Li, J., 2000: Gaussian quadrature and its application to infrared radiative transfer. J. Atmos. Sci., 57, 753-765, https://doi.org/ 10.1175/1520-0469(2000)057<0753:GQAIAT>2.0.CO;2.

- , 2002: Accounting for unresolved clouds in a 1D infrared radiative transfer model. Part I: Solution for radiative transfer, including cloud scattering and overlap. J. Atmos. Sci., 59, 3302-3320, https://doi.org/10.1175/1520-0469(2002)059<3302: AFUCIA $>2.0 . \mathrm{CO} ; 2$.

— , and Q. Fu, 2000: Absorption approximation with scattering effect for infrared radiation. J. Atmos. Sci., 57, 2905-2914, https://doi.org/ 10.1175/1520-0469(2000)057<2905:AAWSEF>2.0.CO;2.

$\ldots$, and H. Barker, 2005: A radiation algorithm with correlated- $k$ distribution. Part I: Local thermal equilibrium. J. Atmos. Sci., 62, 286-309, https://doi.org/10.1175/JAS-3396.1.
— S. Dobbie, P. Raisanen, and Q. Min, 2005: Accounting for unresolved clouds in a 1-D solar radiative-transfer model. Quart. J. Roy. Meteor. Soc., 131, 1607-1629, https://doi.org/ 10.1256/qj.04.31.

Lindner, T. H., and J. Li, 2000: Parameterization of the optical properties for water clouds in the infrared. J. Climate, 13, 1797-1805, https://doi.org/10.1175/1520-0442(2000)013<1797: POTOPF $>2.0 . \mathrm{CO} ; 2$.

Mace, G. G., D. Vane, G. Stephens, and D. Reinke, 2007: Level 2 radar-lidar GEOPROF product: Version 1.0-Process description and interface control document. JPL Rep., 20 pp., http:// www.cloudsat.cira.colostate.edu/sites/default/files/products/ files/2B-GEOPROF-LIDAR_PDICD.P2_R04.20070604.pdf.

Mlawer, E., S. Taubman, P. Brown, M. Iacono, and S. Clough, 1997: Radiative transfer for inhomogeneous atmospheres: RRTM, a validated correlated-k model for the longwave. J. Geophys. Res., 102, 16 663-16 682, https://doi.org/10.1029/97JD00237.

Raisanen, P., H. W. Barker, M. F. Khairoutdinov, J. Li, and D. A. Randall, 2004: Stochastic generation of subgrid-scale cloudy columns for large-scale models. Quart. J. Roy. Meteor. Soc., 130, 2047-2067, https://doi.org/10.1256/qj.03.99.

Stamnes, K., S. C. Tsay, W. J. Wiscombe, and K. Jayaweera, 1988: Numerically stable algorithm for discrete ordinate method radiative transfer in multiple scattering and emitting layered media. Appl. Opt., 27, 2502-2509, https://doi.org/10.1364/ AO.27.002502.

Stephens, G., and Coauthors, 2002: The CloudSat mission and the A-train: A new dimension of space-based observations of clouds and precipitation. Bull. Amer. Meteor. Soc., 83, 17711790, https://doi.org/10.1175/BAMS-83-12-1771.

Tang, G., P. Yang, G. Kattawar, X. Huang, E. Mlawer, B. Baum, and M. King, 2018: Improvement of the simulation of cloud longwave scattering in broadband radiative transfer models. J. Atmos. Sci., 75, 2217-2233, https://doi.org/10.1175/JAS-D-18-0014.1.

Viskanta, R., and M. P. Menguc, 1987: Radiation heat transfer in combustion systems. Prog. Energy Combust. Sci., 13, 97-160, https://doi.org/10.1016/0360-1285(87)90008-6.

von Salzen, K., and Coauthors, 2013: The Canadian Fourth Generation Atmospheric Global Climate Model (CanAM4). Part I: Representation of physical processes. Atmos.-Ocean, 51, 104-125, https://doi.org/10.1080/07055900.2012.755610.

Winker, D., J. Pelon, and M. McCormick, 2003: CALIPSO mission: Spaceborne lidar for observation of aerosols and clouds. Proc. SPIE, 4893, 1-11, https://doi.org/10.1117/12.466539.

Wiscombe, W., 1977: The delta- $M$ method: Rapid yet accurate radiative flux calculations. J. Atmos. Sci., 34, 1408-1422, https://doi.org/ 10.1175/1520-0469(1977)034<1408:TDMRYA > 2.0.CO;2.

Yang, P., K. Liou, L. Bi, C. Liu, B. Yi, and B. Baum, 2015: On the radiative properties of ice clouds: Light scattering, remote sensing, and radiation parameterization. Adv. Atmos. Sci., 32, 32-63, https://doi.org/10.1007/s00376-014-0011-z.

Zhang, F., K. Wu, J. Li, Y. Quan, J.-Q. Zhao, and J. Li, 2016: Analytical infrared delta-four-stream adding method from invariance principle. J. Atmos. Sci., 73, 4171-4188, https:// doi.org/10.1175/JAS-D-15-0317.1. 